\title{
Simplexlike structures of maximally supersymmetric scattering amplitudes
}

\author{
Junjie Rao® \\ Max Planck Institute for Gravitational Physics (Albert Einstein Institute), 14476 Potsdam, Germany
}

(Received 17 July 2017; accepted 14 February 2020; published 16 March 2020)

\begin{abstract}
We elaborate the two-fold simplexlike structures of tree amplitudes in planar maximally supersymmetric Yang-Mills $(\mathcal{N}=4 \mathrm{SYM})$, through its connection to a mathematical structure known as the positive Grassmannian. Exploiting the reduced Grassmannian geometry and the matrix form of on-shell recursion relation in terms of super momentum twistors, we manifest that tree amplitudes can be remarkably refined via the essential building blocks named as fully spanning cells. For a fixed number of negative helicities, an amplitude can be entirely captured by finite, compact information of the relevant fully spanning cells up to an arbitrarily large number of external particles.
\end{abstract}

DOI: 10.1103/PhysRevD.101.061702

\section{INTRODUCTION}

In recent years, enormous progress on scattering amplitudes has been made using various modern approaches beyond Feynman diagrams (see e.g., [1-4] for reviews). In particular, amplitudes of $\mathcal{N}=4 \mathrm{SYM}$ in the planar limit are most understood due to its unmatched symmetries. At both tree and loop levels, dual superconformal invariance manifested by (super) momentum twistors [5], greatly facilitates the calculation of amplitudes and loop integrands in planar $\mathcal{N}=4$ SYM [6]. Explicitly, this is realized by the momentum twistor version of Britto-Cachazo-Feng-Witten $(\mathrm{BCFW})$ recursion relation $[7,8]$, which constructs amplitudes solely from on-shell subamplitudes, eliminating gauge redundancy as well as unphysical internal particles.

In the meanwhile, another unanticipated magic, namely the positive Grassmannian together with on-shell diagrams and decorated permutations [9-11], provides new insights into the on-shell construction of amplitudes. This is mostly achieved in the space of massless spinors, while transforming its entire machinery into momentum twistor space brings extra complexity [12], since each momentum twistor is not characterized by the momentum of its literally corresponding particle, but a kinematic mixture of numerous adjacent particles. It is this entanglement that trivializes momentum conservation, so that we may concentrate on the pure kinematics separated from that universal constraint. However, for nonplanar $\mathcal{N}=4 \mathrm{SYM}$, momentum twistors cannot be defined, while on-shell diagrams still work [13-16], in fact, the broad applicability of on-shell

Published by the American Physical Society under the terms of the Creative Commons Attribution 4.0 International license. Further distribution of this work must maintain attribution to the author(s) and the published article's title, journal citation, and DOI. Funded by SCOAP ${ }^{3}$. diagrams is independent of the number of supersymmetries or spacetime dimensions $[17,18]$.

Back to planar $\mathcal{N}=4 \mathrm{SYM}$, to enhance the advantage brought by positive Grassmannian, we introduce another interesting excursion which brings even more insights and richer structures of amplitudes [19], at tree level for the moment. It is a purely geometric approach working in momentum twistor space without referring to on-shell diagrams and decorated permutations, through establishing the exact correspondence between Grassmannian geometric configurations and Yangian invariants generated by recursion. The momentum twistor $\mathrm{BCFW}$ recursion relation is now presented in the matrix form of positive Grassmannian, which can be nicely deduced from positivity plus a minimal knowledge of momentum twistors. It is a simple linear algebra exercise to read off the geometric configuration from the matrix representative of each $\mathrm{BCFW}$ cell, which can be mapped back to its corresponding Yangian invariant directly. To encode this geometric information more compactly, we need to introduce the reduced Grassmannian geometry for distinguishing linear dependencies of different ranks. In addition, for BCFW cells it is also convenient to denote columns that are set to zero as "empty slots," from which we will later reveal the two-fold simplexlike structures of tree amplitudes, as elaborated in this paper. Then, for a fixed number of negative helicities, an amplitude can be entirely captured by finite characteristic objects called fully spanning cells up to an arbitrarily large number of external particles.

\section{POSITIVE MATRIX FORM OF MOMENTUM TWISTOR BCFW RECURSION RELATION}

To solely work in momentum twistor space, for a tree amplitude one can always first factor out the maximally helicity-violating (MHV) part, and the rest is the desired Yangian invariant we would like to address. The BCFW 


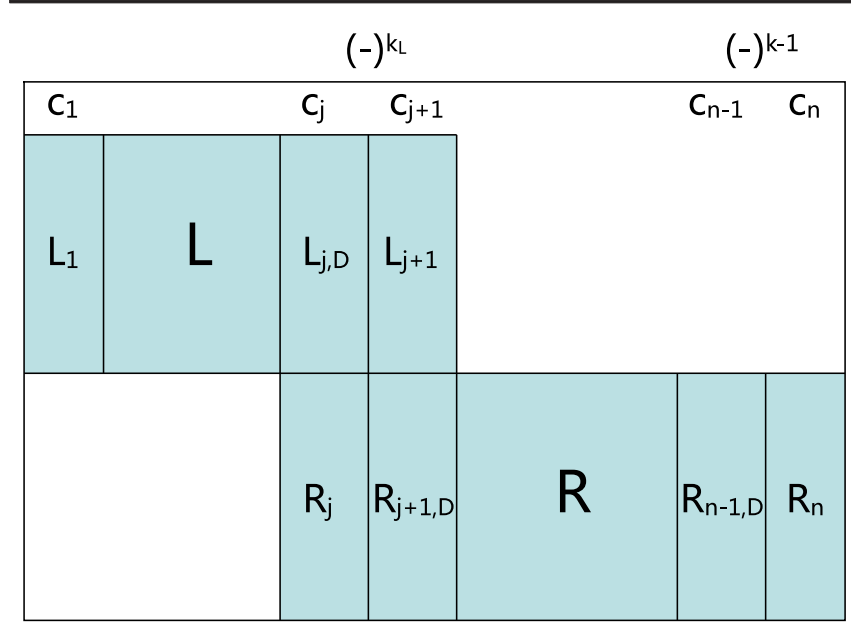

FIG. 1. Positive matrix form of momentum twistor BCFW recursion relation. Sign factors $(-)^{k_{\mathrm{L}}}$ and $(-)^{k-1}$ are associated to the $c_{j}, c_{j+1}$ and $c_{n-1}, c_{n}$ pairs respectively. All the blank regions are filled with zero entries implicitly.

recursion relation starts with the simplest Yangian invariants known as the 5-brackets, then constructs more complex ones repeatedly with certain deformations that impose the on-shell condition of internal particles. From the Grassmannian perspective, this can be deduced from positivity as a consequence of pure geometry. We present the resulting matrix configuration in Fig. 1.

Let us give some explanation. For a $(k \times n)$ matrix $C_{\alpha a}$ to have physical significance, where $(k+2)$ and $n$ are the numbers of negative helicities and total external particles respectively, $C$ has to be positive (all of its ordered minors are positive or zero) and it obeys the orthogonal constraint $C_{\alpha a} Z_{a}=0$ where $Z_{a}$ 's denote $n$ momentum twistors as kinematical data. If we have two such matrices $C_{\mathrm{L}}$ and $C_{\mathrm{R}}$, we can construct a larger one by sewing them in some physical way, which induces deformations of the relevant columns in these two submatrices. To parametrize the deformations, we need an additional row on the top and minimally it has five entries to fulfill the constraint $C_{\alpha a} Z_{a}=0$. In the geometric sense, the physical way above is nothing but imposing positivity of this larger matrix. Explicitly, for $C_{\mathrm{L}}$ spanning from column 1 to $(j+1)$ and $C_{\mathrm{R}}$ from $j$ to $n$ (see Fig. 1), the deformed subcolumns with subscript " $D$ " are given by

$$
\begin{aligned}
L_{j, \mathrm{D}} & =L_{j}+\frac{c_{j}}{c_{j+1}} L_{j+1}, \\
R_{j+1, \mathrm{D}} & =R_{j+1}+\frac{c_{j+1}}{c_{j}} R_{j}, \quad R_{n-1, \mathrm{D}}=R_{n-1}+\frac{c_{n-1}}{c_{n}} R_{n},
\end{aligned}
$$

where the $c$ 's are entries of the top row. To ensure positivity, extra sign factors $(-)^{k_{\mathrm{L}}}$ and $(-)^{k-1}$ must be associated to the $c_{j}, c_{j+1}$ and $c_{n-1}, c_{n}$ pairs respectively. After all $c$ 's find their solutions in $C_{\alpha a} Z_{a}=0$, the matrix above recovers the $\mathrm{BCFW}$ product of Yangian invariants
$[1 j j+1 n-1 n] Y_{\mathrm{L}}(1, \ldots, j, I) Y_{\mathrm{R}}(I, j+1, \ldots, n-1, \hat{n})(2$

where $\mathcal{Z}_{I}=\hat{\mathcal{Z}}_{j+1}=\hat{\mathcal{Z}}_{j}=(j j+1) \cap(n-1 n 1)$ and $\hat{\mathcal{Z}}_{n}=$ $(n-1 n) \cap(1 j j+1)$.

Denoting the matrix in Fig. 1 as $Y_{n-1, j}$, we can express a general tree amplitude (or Yangian invariant, precisely) as

$$
Y_{n}^{k}=\sum_{i=k+3}^{n-1} \sum_{j=2}^{i-2} Y_{i, j} .
$$

Each matrix consists of a subset of BCFW cells of various $k_{\mathrm{L}}$ and $k_{\mathrm{R}}$ satisfying $k_{\mathrm{L}}+k_{\mathrm{R}}=k-1 \geq 0$ and $0 \leq k_{\mathrm{L}, \mathrm{R}} \leq$ $n_{\mathrm{L}, \mathrm{R}}-4$, with $k_{\mathrm{L}}=0$ for $n_{\mathrm{L}}=3$ as the only special case. The "sum" of BCFW cells, or Grassmannian geometric configurations, in fact needs to be specified for avoiding ambiguity of relative signs $[20,21]$. Such a discussion is presented in [19], where we used some linear algebra trick to map BCFW cells back to Yangian invariants, and we plan to give a more systemic treatment in the future. As we will soon see, this literal sum is indirectly justified by the cyclicity of amplitudes via homological identities.

\section{REDUCED GRASSMANNIAN GEOMETRY}

The matrix recursion relation generates more intricate geometric configurations beyond trivial single rows made of five nonzero entries. For example, under the default recursion scheme, the Next-to-Next-to-MHV $\left(\mathrm{N}^{2} \mathrm{MHV}\right)$ $n=7$ amplitude is given by (geometrically this is called a contour)

$Y_{7}^{2}=[7]+[5]+[2]+(23)(45)+(23)(67)+(45)(71)$,

where for instance, [7] is a top cell with the 7th column removed, while (23)(45) denotes vanishing minors $(23)=$ $(45)=0$. These BCFW cells are of $4 k=k(n-k)-2=8$ dimensions, for which kinematic and geometric degrees of freedom are equal (modulo GL $(k)$ invariance and vanishing constraints for the latter).

Representing cells in this way is named as the Grassmannian geometry, and in particular, [i] which denotes the $i$ th column is null, is called an empty slot. Note that, these symbols only make sense when $k, n$ are specified. For $k \geq 3$, we need the reduced Grassmannian geometry. For example, one $\mathrm{N}^{3} \mathrm{MHV} n=9 \mathrm{BCFW}$ cell is

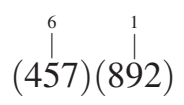

where the "upstairs" parts denote that, columns 5,6 are proportional and so are columns 9,1, while as usual (457) and (892) are $3 \times 3$ vanishing minors. In this way, linear dependencies of different ranks are distinguished unambiguously so that reading off its dimension is transparent. 
We may apply (reduced) Grassmannian geometry to describe the homological identities, which are vanishing relations between a number of "boundary" cells generated by the relevant $(4 k+1)$-dimensional cells. An example is the $\mathrm{N}^{2} \mathrm{MHV} n=7$ identity

$$
\begin{aligned}
0= & \partial(12) \\
= & -[1]+[2]-(12)(34)+(12)(45) \\
& -(12)(56)+(12)(67),
\end{aligned}
$$

note that, we have discarded boundary cells that fail to have kinematical supports of $C_{\alpha a} Z_{a}=0$, which in this case are $(712)_{2}$ (abbreviation of (71)(12), and so forth) and (123) $)_{2}$. But still, we abuse the term "homological" here, while the actual kinematics also matters. ${ }^{1}$

This is the only type of identities of $k=2, n=7$ up to a cyclic shift, and it guarantees the cyclicity of $\mathrm{N}^{2} \mathrm{MHV}$ $n=7$ amplitude via

$$
Y_{7}^{2}-Y_{7,+1}^{2}=-\partial(23)-\partial(56)-\partial(71)
$$

where $Y_{7,+1}^{2}$ is the cyclicly shifted (by +1 ) counterpart of $Y_{7}^{2}$ in (4). Remarkably, the cyclicity of $\mathrm{N}^{2} \mathrm{MHV}$ amplitudes up to any $n$ can be shown in a similar but certainly more complicated way. To manifest it demands the two-fold simplexlike structures of tree amplitudes, which we will immediately exhibit in detail.

\section{TRIANGLELIKE DISSECTION OF GENERAL $\mathbf{N}^{k}$ MHV AMPLITUDES}

Performing the BCFW recursion relation in its matrix form and using the representation of reduced Grassmannian geometry, the two-fold simplexlike structures of tree amplitudes naturally emerge, after some simple observation and refinement. As an appetizer, a general NMHV amplitude in terms of 5-brackets is written as

$$
Y_{n}^{1}=\sum_{i=4}^{n-1} \sum_{j=2}^{i-2}[1 j j+1 i i+1]
$$

now in terms of empty slots, it becomes

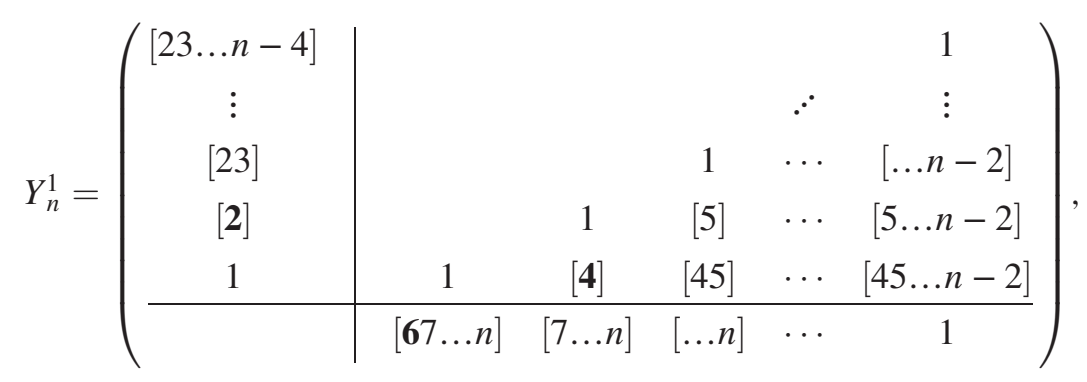

where $Y_{n}^{1}$ is the sum of all entries in the "triangle" above, and each entry is multiplied by its corresponding vertical and horizontal factors, since we have maximally factored out common empty slots to manifest the pattern, which is uniquely determined by the triple $(6,4,2)$ (in bold) for any $n$. This pattern will be later defined as a quadratic growing mode.

The general $\mathrm{N}^{k} \mathrm{MHV}$ amplitude directly follows a similar arrangement of the NMHV triangle, given by

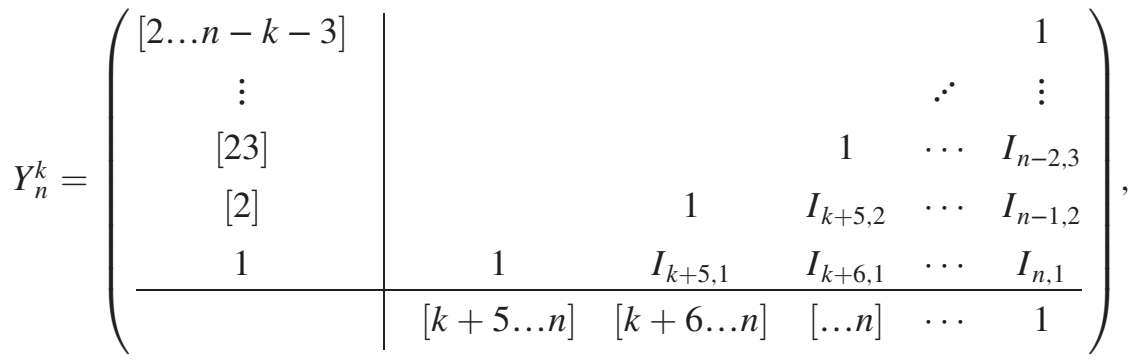

where $I_{i, 1}$ 's in the bottom row, each of which is a sum of BCFW cells, are the only essential objects to be identified, since it is trivial to obtain $I_{i, 1+j}$ by performing a partial cyclic shift $i \rightarrow i+j$ except that label 1 is fixed, for all cells within $I_{i, 1}$. For example, from (4) we already know $I_{7,1}$, then $I_{7,2}$ is simply given by

$$
I_{7,1}=\left(\left\{\begin{array}{l}
(45)(71) \\
{[5]}
\end{array} \quad(23)\left\{\begin{array}{l}
(67) \\
(45)
\end{array}\right) \rightarrow I_{7,2}=\left(\begin{array}{l}
(56)(81) \\
{[6]}
\end{array} \quad(34)\left\{\begin{array}{l}
(78) \\
(56)
\end{array}\right),\right.\right.\right.
$$

\footnotetext{
${ }^{1}$ We thank Jake Bourjaily for pointing out this subtlety.
} 
where $I_{7,1}$ packs up four cells and so does $I_{7,2}$, note the common vanishing minors have been also factored out.

This is the trianglelike dissection of tree amplitudes, which isolates $I_{i, 1}$ for further dissection. Before that, let us digress to discuss how it refines the counting of $\mathrm{BCFW}$ cells. It is known that the number of BCFW terms in tree amplitudes is given by

$$
N_{n}^{k}=\frac{1}{n-3}\left(\begin{array}{c}
n-3 \\
k
\end{array}\right)\left(\begin{array}{l}
n-3 \\
k+1
\end{array}\right) .
$$

The double slicing (vertical and horizontal) in (10) gives its second order difference as

$$
\Delta^{2} N_{n}^{k}=\Delta N_{n}^{k}-\Delta N_{n-1}^{k}=N_{n}^{k}-2 N_{n-1}^{k}+N_{n-2}^{k},
$$

which is exactly the number of BCFW cells in $I_{n, 1}$. For the first nontrivial case $k=2$, we have

$$
\Delta^{2} N_{n}^{2}=(n-5)^{2},
$$

this will be useful as a highly nontrivial consistency check of the further simplexlike structure.

\section{N²MHV FULLY SPANNING CELLS AND SOLID SIMPLICES}

Further dissecting $I_{i, 1}$ reveals the following pattern:

$$
\begin{aligned}
I_{i, 1}= & \left(\text { cells descend from } I_{i-1,1}\right) \\
& +(\text { new fully spanning cells for } n=i),
\end{aligned}
$$

the first part of cells above follow simple patterns of the solid simplices, and so do the second when $i$ is increased by one or more. The fully spanning cells are named such that none of their $i$ columns are removed when they first show up in $I_{i, 1}$. Each time we increase $i$ by one, they are the only objects need to be identified together with their growing parameters, which will uniquely determine their "growing" patterns in $I_{n, 1}$ up to any $n$. Remarkably, the recursive growth of new fully spanning cells terminates at $n=4 k+1$, as we will later see.

Explicitly, let us illustrate this pattern of $I_{i, 1}$ for the family of $\mathrm{N}^{2} \mathrm{MHV}$ amplitudes, their fully spanning cells are given by

$$
\begin{aligned}
G_{7,0} & =\left\{\begin{array}{cc}
(45)(71) \\
{[5]}
\end{array} \quad(5)\right. \\
G_{7,1} & =(23) \begin{cases}(67) & (6,4) \\
(45) & \end{cases} \\
G_{8,1} & = \begin{cases}(234)_{2}(678)_{2} & (7,4) \\
(456)_{2}(781)_{2} & (7,5) \\
(23)(456)_{2}(81) & (6,4)\end{cases} \\
G_{9,2} & = \begin{cases}(2345)_{2}(6789)_{2} \\
(23)(4567)_{2}(891)_{2}\end{cases}
\end{aligned}
$$

where $G_{i, m}$ is the part purely made of fully spanning cells in $I_{i, 1}$ and $m$ is its corresponding growing mode, followed by their growing parameters (some cells share the same parameters). Note that [5] in $G_{7,0}$ above actually originates from $I_{6,1}$ as a top cell, but for convenience it is put together with (45)(71) as they share one parameter.

The meaning of growing modes and parameters can be seen from, for instance, how three sample cells below of constant, linear and quadratic modes mutate as $i$ of $I_{i, 1}$ increases, according to

$$
\begin{aligned}
(45)(71) \rightarrow[5](46)(81) & \rightarrow[56](47)(91), \\
(23)(67) \rightarrow[6](23)(78) & \rightarrow[67](23)(89) \\
+[4](23)(78) & +[47](23)(89) \\
& +[45](23)(89),
\end{aligned}
$$

for $I_{7,1}, I_{8,1}$ and $I_{9,1}$, and

$$
\begin{aligned}
(2345)_{2}(6789)_{2} \rightarrow[8](2345)_{2}(67910)_{2} & \rightarrow[89](2345)_{2}(671011)_{2} \\
+[6](2345)_{2}(78910)_{2} & +[69](2345)_{2}(781011)_{2} \\
+[4](2356)_{2}(78910)_{2} & +[67](2345)_{2}(891011)_{2} \\
& +[49](2356)_{2}(781011)_{2} \\
& +[47](2356)_{2}(891011)_{2} \\
& +[45](2367)_{2}(891011)_{2},
\end{aligned}
$$

for $I_{9,1}, I_{10,1}$ and $I_{11,1}$. Note the increasing numbers of empty slots induce partial cyclic shifts for the associated cells, while maintaining their cyclic topologies, similar to that of obtaining $I_{i, 1+j}$ from $I_{i, 1}$ in (10). We can further extract the key mathematical objects that best describe all such patterns, namely the solid simplices.
A solid $m$-simplex is fully characterized by its growing mode $m,(m+1)$ growing parameters and level which counts the empty slots at each point within it. The term "solid" means inside the simplex there are also a number of points. In Fig. 2, we depict three solid simplices of constant, linear and quadratic growing modes $(0-, 1-$, 
8910
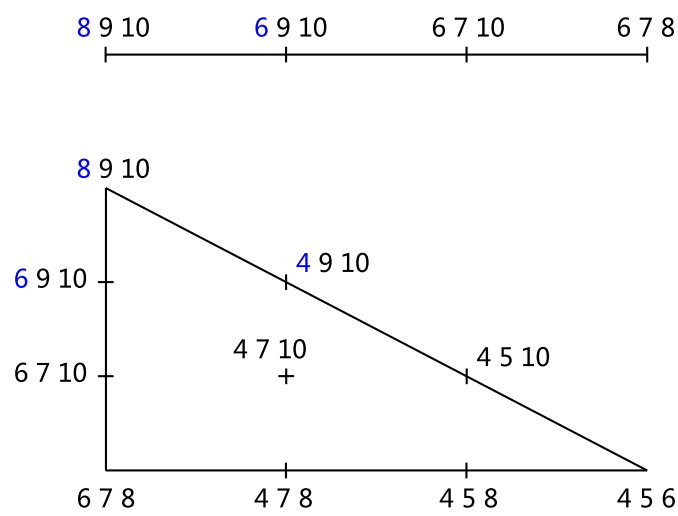

FIG. 2. Solid 0-, 1-, 2-simplices up to level 3, of growing parameters $(8),(8,6),(8,6,4)$ respectively.

and 2-modes for short) up to level 3, of growing parameters $(8),(8,6),(8,6,4)$ respectively.

Remarkably, the 2-mode with parameters $(6,4,2)$ exactly characterizes the NMHV triangle (9), which is extended to the general $\mathrm{N}^{k} \mathrm{MHV}$ trianglelike dissection in (10). Obviously, the two-fold simplexlike structures are closely related, and the pattern of a 2-mode is manifest in the form which maximally factors out empty slots as (9). General solid $m$-simplices similarly follow these patterns [19]. Now we can only concentrate on empty slots, while the induced geometric configurations can be trivially inferred from their original cyclic topologies.

Let us immediately see the power of solid simplices for the $\mathrm{N}^{2} \mathrm{MHV}$ case: back to (16), according to the growing modes and levels of fully spanning cells, for any $n$, it is easy to count the terms in $I_{n, 1}$ as

$2+2(n-6)+3(n-7)+2 \cdot \frac{(n-7)(n-8)}{2}=(n-5)^{2}$,

which nicely matches $\Delta^{2} N_{n}^{2}$ in (14).

\section{TERMINATION OF THE RECURSIVE GROWTH OF FULLY SPANNING CELLS}

Naturally, it is economical to only generate the fully spanning cells along with their growing parameters, for a given $k$. This is called the refined $\mathrm{BCFW}$ recursion relation [19], which constructs fully spanning cells solely from those of lower $k$ 's, and it terminates at $n=4 k+1$. Explicitly, we find the numbers of fully spanning cells for $k=1,2,3$ as summarized in the table below (all the unspecified entries are zeros implicitly).

\begin{tabular}{lllllllllll}
\hline & \multicolumn{10}{c}{$k$} \\
\cline { 2 - 10 }$n$ & 5 & 6 & 7 & 8 & 9 & 10 & 11 & 12 & 13 & 14 \\
\hline 1 & 1 & & & & & & & & & \\
2 & & 1 & 3 & 3 & 2 & & & & & \\
3 & & & 1 & 7 & 18 & 27 & 26 & 15 & 5 & \\
& & & & & & & & & &
\end{tabular}

Note the first fully spanning cell for any $k$ is a top cell, and it is the only one in the anti-MHV sector $(n=k+4)$.

\section{SUMMARY AND OUTLOOK}

So far we have witnessed the concise profile of tree amplitudes in planar $\mathcal{N}=4 \mathrm{SYM}$, with the aid of the matrix form of BCFW recursion relation and reduced Grassmannian geometry. The two-fold simplexlike structures are an extension following the same logic of, e.g., [22-24]. It is the simple Parke-Taylor formula [25] of MHV tree amplitudes of gluons that first freed us from countless Feynman diagrams, and up to this point, the similar idea has been extended to the solid simplices for general $\mathrm{N}^{k} \mathrm{MHV}$ amplitudes from the Grassmannian perspective, so that infinite terms now can be essentially captured by finite, compact information.

In the future, we will present how this formalism helps manifest the cyclicity of general $\mathrm{N}^{k} \mathrm{MHV}$ amplitudes. The NMHV sector has been solved in [19], so the first nontrivial case is the $\mathrm{N}^{2} \mathrm{MHV}$ sector. Also, we would like to explore how to extend it to, say, the 1-loop integrand level, which is expected to be much more intricate.
[1] L. J. Dixon, arXiv:hep-ph/9601359.

[2] F. Cachazo and P. Svrcek, Proc. Sci. RTN2005 (2005) 004 [arXiv:hep-th/0504194].

[3] J. M. Henn and J. C. Plefka, Scattering Amplitudes in Gauge Theories (Springer, New York, 2014).

[4] H. Elvang and Y.-t. Huang, Scattering Amplitudes in Gauge Theory and Gravity (Cambridge University Press, Cambridge, England, 2015).
[5] A. Hodges, J. High Energy Phys. 05 (2013) 135.

[6] N. Arkani-Hamed, J. L. Bourjaily, F. Cachazo, S. Caron-Huot, and J. Trnka, J. High Energy Phys. 01 (2011) 041.

[7] R. Britto, F. Cachazo, and B. Feng, Nucl. Phys. B715, 499 (2005).

[8] R. Britto, F. Cachazo, B. Feng, and E. Witten, Phys. Rev. Lett. 94, 181602 (2005). 
[9] N. Arkani-Hamed, F. Cachazo, C. Cheung, and J. Kaplan, J. High Energy Phys. 03 (2010) 020.

[10] N. Arkani-Hamed, F. Cachazo, and C. Cheung, J. High Energy Phys. 03 (2010) 036.

[11] N. Arkani-Hamed, J. L. Bourjaily, F. Cachazo, A. B. Goncharov, A. Postnikov, and J. Trnka, Grassmannian Geometry of Scattering Amplitudes (Cambridge University Press, Cambridge, England, 2016).

[12] Y. Bai and S. He, J. High Energy Phys. 02 (2015) 065.

[13] N. Arkani-Hamed, J. L. Bourjaily, F. Cachazo, A. Postnikov, and J. Trnka, J. High Energy Phys. 06 (2015) 179.

[14] S. Franco, D. Galloni, B. Penante, and C. Wen, J. High Energy Phys. 06 (2015) 199.

[15] B. Chen, G. Chen, Y. K. E. Cheung, R. Xie, and Y. Xin, Eur. Phys. J. C 78, 164 (2018).

[16] J. L. Bourjaily, S. Franco, D. Galloni, and C. Wen, J. High Energy Phys. 10 (2016) 003.
[17] H. Elvang, Y.t. Huang, C. Keeler, T. Lam, T. M. Olson, S. B. Roland, and D. E. Speyer, J. High Energy Phys. 12 (2014) 181.

[18] P. Benincasa and D. Gordo, J. High Energy Phys. 11 (2017) 192.

[19] J. Rao, J. High Energy Phys. 12 (2017) 147.

[20] J. L. Bourjaily, arXiv:1212.6974.

[21] T. M. Olson, J. High Energy Phys. 08 (2015) 120.

[22] J. M. Drummond and J. M. Henn, J. High Energy Phys. 04 (2009) 018.

[23] N. Arkani-Hamed, J. Bourjaily, F. Cachazo, and J. Trnka, J. High Energy Phys. 01 (2011) 049.

[24] J. L. Bourjaily, J. Trnka, A. Volovich, and C. Wen, J. High Energy Phys. 01 (2011) 038.

[25] S. J. Parke and T. R. Taylor, Phys. Rev. Lett. 56, 2459 (1986). 